\title{
Characterization of materials for a vacuum-ultraviolet polarization analyzer
}

Corti, Gianni (Dipartimento di Astronomia e Scienza dello Spazio, Università di Firenze, Largo Fermi 2, 50125 Florence, Italy); Romoli, Marco (Dipartimento di Astronomia e Scienza dello Spazio, Università di Firenze, Largo Fermi 2, 50125 Florence, Italy)

Applied Optics, Volume 42, Issue 19, pp. 3950-3956 (2003).

Published in Jul 2003

DOI: 10.1364/AO.42.003950

Astronomical measurements in the vacuum-UV spectral region $(30-190 \mathrm{~nm})$ require space instruments and techniques that are at the boundary between visible and $\mathrm{x}$-ray techniques. In vacuum-UV polarimetry, transmission polarizers must be replaced by reflection polarizers because of the lack of transparent materials in the 30-105-nm range. The general features of a single reflection polarization analyzer for the vacuum UV are introduced and described, with particular emphasis on astrophysical applications. In particular, we discuss the trade-off conditions for optimum polarization and throughput of a single-reflection surface in the vacuum UV, introducing a quality factor parameter. The polarization performances of various reflecting materials are obtained with a reflecting vacuum-UV polarization analyzer laboratory model designed and built to measure the state of linear polarization. On the basis of a comparison of the quality factors, calcium fluoride is determined to be the best-performing material. Finally, we discuss the laboratory polarimetric characterizations of the material properties for astronomical application of the polarization analyzer.

\section{Keywords:}

optical polarisers, polarimetry, ultraviolet spectra, ultraviolet spectroscopy, angular measurement 